\title{
Customized E-learning Mobile Application with Student Involvement in 2016
}

\author{
Piya Techateerawat \\ Engineering Faculty, Thammasat University, Thailand
}

\begin{abstract}
The new trend of E-learning is approaching the users. As digitalized data is a mandatory tool for education, the conventional E-learning tools are not up to the user expectation. In a large scale of organization also needs to invest a large cost for modify the tools to fit the organization culture. These challenges limit the small and medium size organization to have its own customized E-learning system. This research is proposed the customized E-learning system in the IT-ready organization. This paper shows alternative method by developing E-learning system based on Android and PHP framework system. To evaluate the impact of the proposed system, sampling of Thai high school that is future student of engineering faculty, Thammasat University in 2016 is chosen for this research. As a result, standard Android framework can be alternated solution for customized Elearning system. Also, the developing cost is managed in the organization plan as well as gaining more user involvement in E-learning activities because of the customized purpose, culture, flow and contents.
\end{abstract}

Keywords: E-learning, Android, Customized System.

\section{Introduction}

Electronic learning (E-learning) is assisting the education in school and organization to deliver knowledge in electronic form. The main objective is to follow the trend of immediate data retrieve and sharing. However, many existing E-learning system is in the static form where the trend of users are changing with social media and high-speed communication. The expectation of users also changes to obtain digested information when their need. Also, the knowledge is expected to be rapid easy and digested information for learners.

This paper shows the alternative solution that each own organization developed its own E-learning system by using the general system. The system is also expected to be able to customize to suit their own organization's purpose, culture, objective and member needs.

\section{E-Learning System}

E-learning is a system based on web-enabling technologies. The purpose of system is to provide the education contents for learners. An easy access support remote learning and extra-hour learning. E-learning is cover in academic, corporate and consumer fields that have been exceed $\$ 2$ trillion worldwide since 2001[1, 2].

The main strategy is tended to improving the quality of learning; access to education and training; reducing the cost of education; and improving the cost-effectiveness of education [3]. So, E-learning services are developed as $24 \times 7$ online, live mentors, real-time chat forums, extensive continually updated database, dynamic test assignment, real-time response and training materials.

A framework is also important for success of E-learning course. The initiative of E-learning is focus on a vision for institution, development of a technology development plan, and maintenance of a reliable technology, technology support to staff and students and provision of time that engaged in E-learning developments [4-5].

The cost of E-learning is increased 83 percent annual growth rate in 1998-2003 [6]. The scale of E-learning is estimated to be one of the largest sectors in the world [7]. For developing country e.g. Thailand, the cost of 
commercial package is the main issue as well as the maintenance cost. However, the open source is strictly on fundamental scheme and required advanced programmer to adapt or customize the package.

\section{Framework}

PHP framework is a structure that prepare for programmer to develop web application. PHP is one of the programming languages that design for web application. This assist for both user and server side to communicate and interact and share contents over the network. Most of the PHP framework is based on ModelView-Controller, this scheme let programmer customize the framework to meet the requirement instead of the writing the source code from beginning. Also, most of the framework is based on open-source, so it can minimize the cost of license and maintenance.

The advantage of using PHP framework is let the structure more robust and more secure from the prepared package compared to developing from bare-bone. In addition, the framework has prepared the libraries and themes for developer to choose and apply. So, implementation can be rapid and more convenience to meet the requirement.

Content Management System (CMS) is also one of the benefits that can be implemented from PHP framework. This system assists the web application to organize the content for appropriate users and services.

The special request is also to add the gaming features. The project designs the main character model of the game in 3D by using Blender. Next, items are created and developed in 2D and 3D game by using Unity3D. This game contains many scenes such as Main Scene, Menu Scene, Game Scene etc., and the application also includes the question scene.

The gaming section in E-learning system is chosen Unity3D as a main 3D structure that can be cooperative with Android system. The advantage of this framework is to develop the model in 3D and can blend the model with 2D pictures. Also, all the main feature can be operated in Android system.

\subsection{Planning \& Development}

This E-learning system is based on the actual case from Thai high school that is future student of engineering faculty, Thammasat University in 2016. The development and evaluation is also based on the actual activity that interacts with students.

The design must be compatible to the new mobile devices (e.g. smart phone and tablets). The contents in each screen should be simplified with one main idea.

The purpose of this E-learning system is to support the actual class and student has sufficient knowledge in using computer technology. The requirement and design of this system is survey from in-class students.

\subsection{Requirement}

- The system must be convenient for in-class student to apply and access.

- Students which do not enroll may apply and register for the interest class.

- Every user can browse the overview of all subjects.

- Only lecturers and applied students can access to the forum.

- Search feature allow everyone to search all contents in every subjects.

- Students may request additional topics of contents.

- The system may suggest the subject and contents that related for each person.

- The system must keep history to activity and test result for each student.

- Test and Quiz can be added in every subject from lecturers.

- Each content can be "Like" and "Share" from Facebook.

- Calendar is allowing each person to add the activity in the system.

- "My Favorite" allows every user to add/modify/delete items.

- Fun/Entertain/Game section 


\subsection{Game Design}

To create games on the Android operating system with Unity3D version 5, starting from importing the model from Blender and coding to control the movement of a model. After that, importing the designed wallpaper and create a game. The game has several scenes: Main Scene is the first scene after open this game. When player tap a "play" button, game will change Menu Scene. Player must select 1 of 4 states to play. At the first state have story video before the game is started for player have motivation. Therefore, this project has Question Scene at the end of each state for player get knowledge and interest

Our developing steps, we design a story board of the game. And present to users. And then we design scenario and rules of the game. After that we design User Interface, scene and background of the game.

Developers plan the game to start at Main Scene with play button. When player tap play button, the game will go to Menu Scene. In this scene, player can select the section to play. This game is a running game with item collection. Each state will be several obstacles with many difficulty levels. And at the end of state, player must answer the question about Software Engineering. If player answer correct, player can pass to next state. If player answer incorrect, player must go back to play this state again.

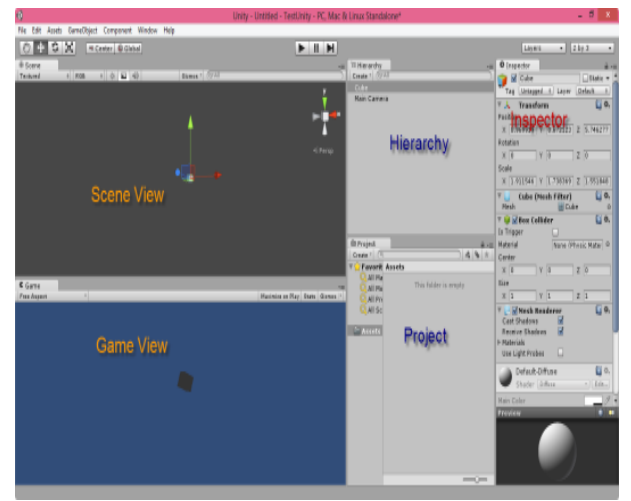

Fig. 1. Unity 3D Application
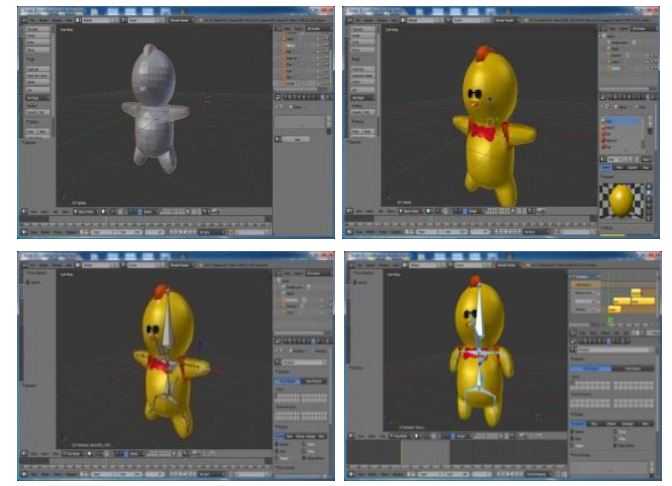

Fig. 2. Main 3D characters model

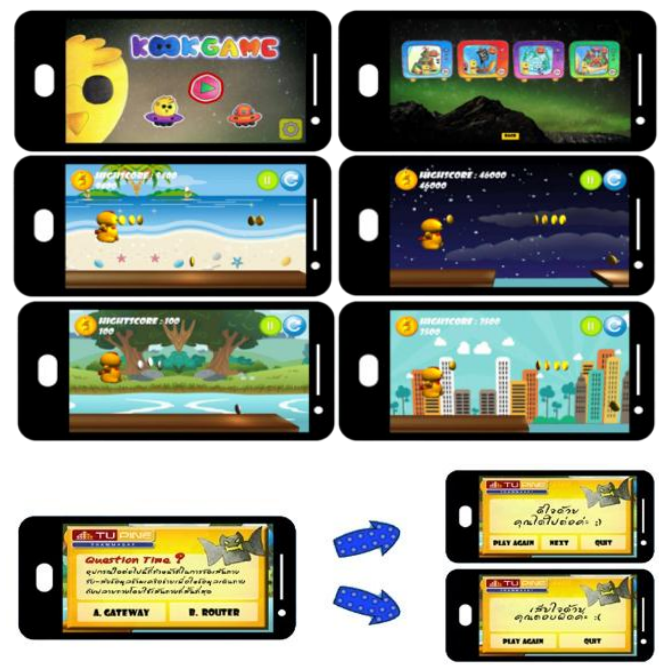

Fig. 3. Game Display

\section{Result}

The end result of system is created based on the design, implementation and testing. The overview of final result is operated by the following features: 
- Log-in to the system.

- Registering to the system.

- Access to E-learning contents \& downloadable contents.

- "Like" of specific content to share on Facebook.

- Some E-learning subject provide optional quiz.

- Search function.

- Forum for learner to share/discuss knowledge.

- File organization Add, Modify, Delete.

- Profile management.

- History organization (Review Content).

- The system may suggest popular/new/related contents based on each person.

In game section, a game application is done. This game is work on Android operating system efficiently. This project made developers have knowledge about coding game application on Android operating system and use to promote to people who are interested in the related knowledge. The developers bring game to trial with the trial players found that 96 percent is satisfied. These results represent that this game makes players have gaining knowledge in the relaxed and entertain area.

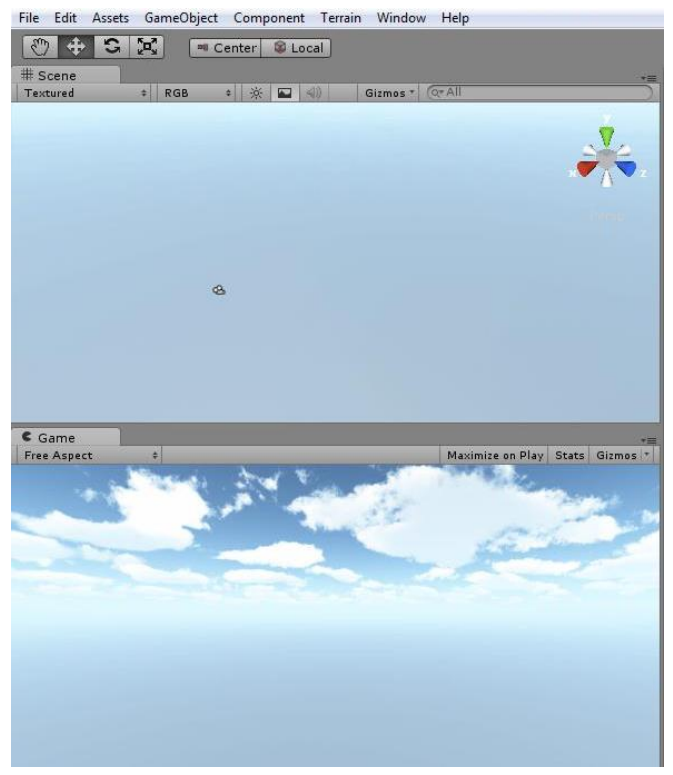

Fig. 4. 2D blending in gaming section.

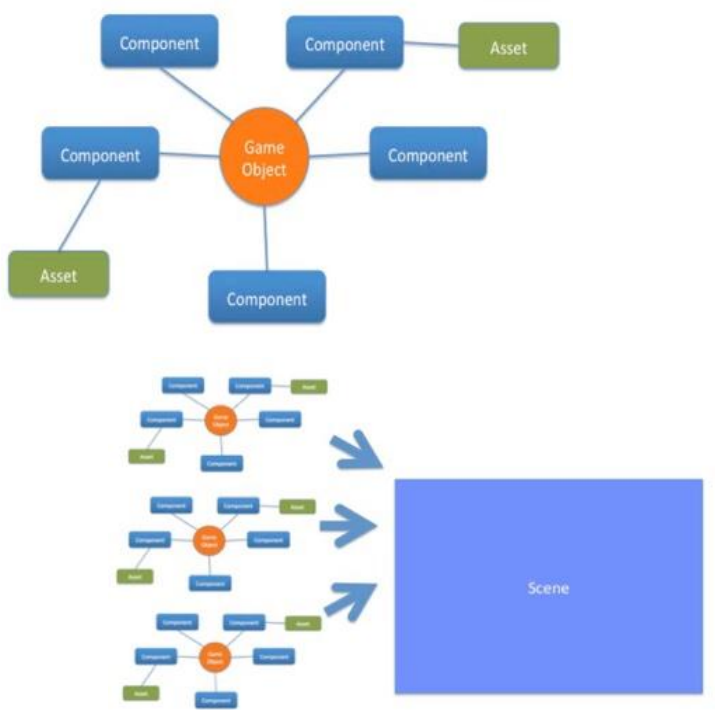

Fig. 5. GameObject: To design in each scenario and combine to a final Scene.

\section{Discussion}

As this system is created based on the actual user (high school students) requirement and reviewed by the same group student, our purpose is to gain more contribution of student. The objective is gain more attention and support the in-class education.

As shown in Table 1, our objective is can be achieved by let the users to customize their own E-learning system. The success area is shown in number of users that use the updated system and return frequently. Also, the supportive in game section can evaluate the interest in high school students. This section is added to the proposed system and is the significant support factor for users to share the social media as well as asked friends to attend on this E-learning system.

However, the gaining of interested and more data to use in this application need more data traffic to communicate between the users and system. In our proposed system, there is no lockage in this scheme. 
However, the organization with the limited resource may need to prepare the computation resource, network resource, bottle neck communication and support team for the proposed system.

Overall, the customized system shows the significant improved in main objective of E-learning system. This also provides the solution that new trend of users need the information in their own need format and their own need. The alternative solution is served their task achieved in this area.

\section{Conclusion}

E-learning is challenged in every organization to customize for appropriate for each organization. The difference in objective, members' background and knowledge leads to different expectation. The customization system is a solution with a high cost of out-of-the-box software.

This paper shows alternative method by developing E-learning system based on PHP framework that focus the designed for mobile users. This proposed also added the game section as the users in the high school level. The entertain and meet their expectation can deliver high satisfaction and active use in their own design.

The trend of user involvement is improving the participation in most of area especially comparing the result of 2105 and the earlier year.

TABLE 1: Survey Result

\begin{tabular}{|c|c|c|}
\hline Topic & Old System & $\begin{array}{c}\text { New } \\
\text { System }\end{array}$ \\
\hline Percentage of student accessing & $8 \%$ & $92 \%$ \\
\hline Percentage of returning user & $30 \%$ & $85 \%$ \\
\hline Satisfaction of game section & $0 \%$ & $98 \%$ \\
\hline Total bandwidth use per class & $450 \mathrm{MB}$ & $15 \mathrm{~GB}$ \\
\hline $\begin{array}{c}\text { Satisfaction rate from user } \\
\text { (100\%-Excellent, 0- need to improve) }\end{array}$ & $40 \%$ & $95 \%$ \\
\hline Number of active activities per class & 0.5 & 22 \\
\hline
\end{tabular}

\section{Acknowledgements}

We would like to thank Faculty of Engineering, Thammasat University, Thammasat University, Optical and Quantum Communication Research Lab and National Research Council of Thailand.

\section{References}

[1] Cisco Systems, Cisco Systems IQ Atlas, Cisco, 2001.

[2] K. Fry, "E-learning markets and providers: some issues and prospects", Education + Training, Vol. 43 No. $4 / 5$ pp. 233 9, 2001. https://doi.org/10.1108/EUM0000000005484

[3] S.M. Furnell, P.D. Onions, U. Bleimann, U. Gojny, M. Knahl, H. F. Roder and P.W. Sanders, "A security framework for online distance learning and training", Internet Research: Electronic Networking Applications and Policy, Vol. 8 No. 3, pp. 236-42, 1998. https://doi.org/10.1108/10662249810217821

[4] S. Alexander, "E-learning developments and experiences", Education + Training, Vol. 43 No. 4/5 pp. 240-8, 2001. https://doi.org/10.1108/00400910110399247

[5] S.H. Garrison and D.J. Borgia, "Using an Internet based distance learning to teach introductory finance", Campus-Wide Information Systems, Vol. 16 No. 4, pp. 136-9, 1999. https://doi.org/10.1108/10650749910289892

[6] D. Lance, “ Venture captial veiwpoints and E-learning futures”, The Business of E-learning: Bringing Your Organizationin the Knowledge Economy, Univeristy of Technology Sydney, 2000.

[7] P. Henry, "E-learning Technology, Content and Services", Education + Training, Vol. 43 No. 4, pp. 249-55, 2001. https://doi.org/10.1108/EUM0000000005485 